\title{
Détermination des rayons de Céphéides ${ }^{\star, \star \star}$
}

\author{
V. Vitesses radiales et dimensions de 22 Céphéides galactiques \\ Determination of the radii of Cepheids \\ V. Radial velocities and dimensions of 22 galactic Cepheids
}

\author{
M. Imbert \\ Observatoire de Marseille, 2 place Le Verrier, F-13248 Marseille Cedex 04, France
}

Reçu le 7 avril ; accepté le 9 septembre 1999

\begin{abstract}
We present radial velocity data for 22 galactic Cepheid stars obtained with Coravel spectrometer. Continuous observation over several years has enabled us to obtain 852 individual velocities covering all the phases of the pulsation. The mean number of measurements per star is 39, ranging from 20 to 113 . For each star radial velocity versus phase diagrams have been fitted by analytical relation, and the stellar radius variation has been derived by integration of this relation over the whole period. Using recent $u b v$ photometry of the literature and velocity curves, we have calculated the radii of the stars using a method based on the Baade-Wesselink concept. For these 22 Cepheids we give a linear logarithmic periodradius relation with a range of 2,4 to 45 days.
\end{abstract}

Key words: stars: cepheids — stars: fundamental parameters - techniques: radial velocities

\section{Introduction}

La détermination des rayons des Céphéides est essentielle pour deux raisons. Elle donne tout d'abord des informations fondamentales concernant l'évolution stellaire, et ensuite, elle est la voie la plus sûre pour évaluer les distances. Grâce à la grande luminosité intrinsèque de ces étoiles, les distances peuvent être déterminées aussi bien à l'intérieur qu'à l'extérieur de la Galaxie. Dans ce contexte, il est très intéressant de pouvoir disposer d'une relation, la plus sûre

Send offprint requests to: M. Imbert

* Les observations ont été effectuées à l'Observatoire de Haute-Provence (CNRS).

** Le tableau 1 est disponible seulement sous forme électronique au CDS via ftp à cdsarc.u-strasbg.fr (130.79.128.5) ou via http://cdsweb.u-strasbg.fr/Abstract.html possible, liant le rayon à la période. Les algorithmes de calcul de ces rayons sont essentiellement basés sur le concept de Baade-Wesselink, impliquant l'analyse simultanée des vitesses radiales, des luminosités et des indices de couleur tout au long du cycle de pulsation.

\section{Observations}

Le spectromètre à corrélation Coravel a été mis en service sur le télescope suisse d'un mètre de l'Observatoire de Haute-Provence en 1977 (Baranne et al. 1979). Dès 1978 nous avons entrepris la mesure systématique des vitesses radiales, à toutes les phases de la pulsation, de nombreuses Céphéides galactiques. Cette étude a pour but de déterminer précisément les dimensions de ces étoiles et conséquemment l'étalonnage de la relation période-rayon pour ces objets. Ce travail présente les vitesses radiales couvrant toutes les phases de la pulsation pour 22 étoiles de ce programme. Ces 852 mesures individuelles ont été acquises durant 20 ans d'observation. Nous avons obtenu, en moyenne, 39 vitesses par étoile, les extrêmes étant 20 et 113. L'erreur moyenne sur la détermination de la vitesse est de $0,55 \mathrm{~km} \mathrm{~s}^{-1}$, tandis que le résidu moyen relativement à l'ajustement est de $0,64 \mathrm{~km} \mathrm{~s}^{-1}$. Les observations sont listées dans le tableau 1.

\section{Représentation analytique de la pulsation}

Nous avons montré précédemment (Imbert et al. 1985, 1989) que le mouvement radial de pulsation pouvait être décrit par une expression analytique de la forme :

$V_{\mathrm{r}}(t)=V_{\mathrm{o}}+\sum_{m} K_{m}\left(e_{m} \cos \omega_{m}+\cos \left(\nu_{m}(t)+\omega_{m}\right)\right)$

$\nu_{m}$ étant défini par :

$\operatorname{tg}\left(\nu_{m}(t) / 2\right)=\left[\left(1+e_{m}\right) /\left(1-e_{m}\right)\right]^{1 / 2} \operatorname{tg}\left(E_{m} / 2\right)$ 


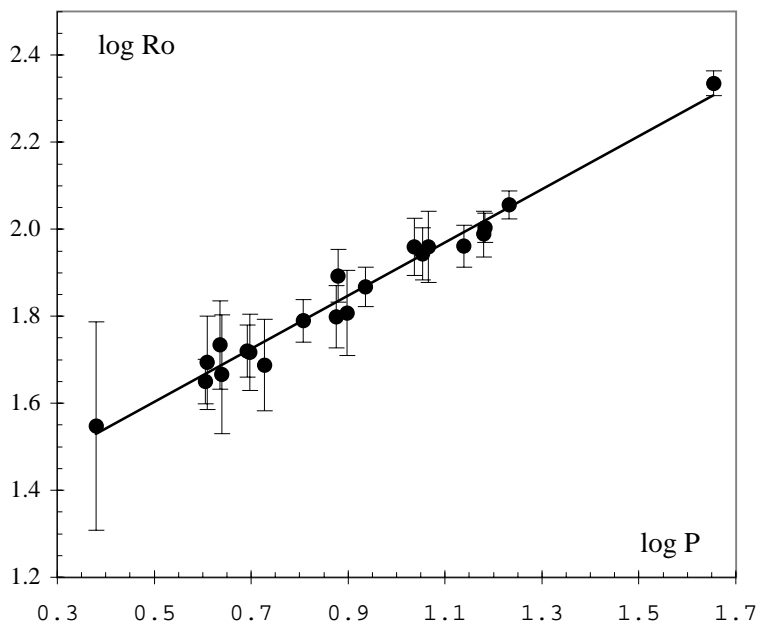

Fig. 1. Relation entre $\log R_{\mathrm{o}}$ et $\log P$. L'étoile BL Her n'est pas représentée

avec $E_{m}-e_{m} \sin E_{m}=M_{m}$

et $M_{m}=2 \pi\left(t-T_{o_{m}}\right) / P_{m}$

$t$ est l'époque d'observation et $P_{m}$ la période du mouvement $m$.

La valeur de m est un pour les Céphéides sans "bump", deux pour les Céphéides avec "bump", et, exceptionnellement trois quand deux mouvements ne suffisent pas à représenter correctement les observations (BL Her). Cet ajustement permet de représenter objectivement les observations de vitesse radiale en évitant les oscillations parasites introduites par un ajustement par développement de Fourier. Il permet en outre de maîtriser le nombre d'extremums de la courbe et donne une très bonne représentation de la pulsation, même dans le cas de mesures peu nombreuses ou mal réparties en phase. Toutes les formes de courbes rencontrées dans ce travail sont parfaitement représentées par ce lissage (Figs. 2, 3 et 4). Cette représentation analytique de la vitesse radiale de l'étoile au cours de sa pulsation est formellement identique à celle décrivant la variation de vitesse radiale des composantes d'un système double spectroscopique. On peut ainsi assimiler la variation de vitesse radiale d'une Céphéide à une somme de mouvements képlériens fictifs (Imbert et al. 1985; Imbert 1987; Imbert et al. 1989). Dans le cas de Céphéides doubles spectroscopiques, ces mouvements fictifs s'ajoutent au mouvement képlérien réel dû à la duplicité (Imbert 1994; Imbert 1996).

Les paramètres de lissage à calculer, $V_{\mathrm{o}}, e_{m}, \omega_{m}, K_{m}$ et $T_{\mathrm{O}_{m}}$, sont au nombre de 5,9 ou exceptionnellement 13, auxquels s'ajoutent éventuellement une, deux ou trois valeurs de $P_{m}$. $V_{\mathrm{o}}$ est la vitesse du centre de masse de l'étoile et $K_{m}$ l'amplitude de la composante $m$ du mouvement. $e_{m}$ et $\omega_{m}$ doivent être considérés comme des paramètres de forme. $T_{\mathrm{o}_{m}}$ est l'époque de passage au pé- riastre de l'orbite fictive, mais ne correspond pas, en général, à un instant remarquable dans la cycle de pulsation. Le calcul de ces éléments orbitaux fictifs ou réels se fait par la méthode classique par améliorations itératives des éléments approchés de départ. Tous les calculs relatifs aux recherches de périodes, détermination des éléments orbitaux approchés et amélioration de ceux-ci par la méthode de moindres carrés de Schlesinger (1908), ont été effectués avec le programme SB5MCEX écrit en VBA Excel et mis en œuvre sur Power Macintosh. Ce programme, très interactif, permet de rechercher les périodes (Imbert 1972), de calculer des éléments approchés par une variante numérique de la méthode graphique de LehmanFilhès (1894), et de déterminer les éléments orbitaux définitifs par itérations successives. Dans ce programme, il est possible de prendre en compte jusqu'à cinq mouvements képlériens superposés. Le programme donne en outre la possibilité de fixer n'importe quel élément à une valeur prédéterminée, comme la période ou l'excentricité $e$. Les éléments orbitaux fictifs calculés par la méthode ci-dessus sont donnés dans le tableau 2. Les différences, $\mathrm{O}-\mathrm{C}$, entre la valeur observée et la valeur calculée avec l'ajustement figurent dans le tableau 1.

\section{Calcul des dimensions}

\subsection{Amplitude de variation des rayons}

L'effet Doppler observé, dû au déplacement radial de l'atmosphère de l'étoile, n'est pas exactement la vitesse d'expansion $V_{\mathrm{e}}$, mais la moyenne des vitesses radiales intégrée sur tout l'hémisphère visible. La vitesse d'expansion est évidemment la seule à considérer pour le calcul du déplacement. L'intégration sur tout le disque conduit à une relation linéaire liant la vitesse d'expansion à la vitesse radiale observée $V_{\mathrm{e}}(t)=-\alpha V_{\mathrm{r}}(t)$, où $\alpha$ et le facteur de projection (Imbert 1981a). Pour Coravel, la valeur de $\alpha$ retenue est 1,36 (Burki et al. 1982; Burki \& Benz 1982).

L'amplitude de variation du rayon de l'étoile sera donnée par

$\Delta R=\left|\alpha \int_{t_{2}}^{t_{1}} V_{\mathrm{r}}(t) \mathrm{d} t\right|$

où $t_{1}$ et $t_{2}$ sont les deux instants du cycle de pulsation où le rayon est extrémal, c'est-à-dire quand $V_{\mathrm{r}}(t)=V_{\mathrm{o}}$. La théorie du mouvement dans un système double spectroscopique, exposée, notamment par Binnendijk (1960), montre que

$Z(t)=\int V_{\mathrm{r}}(t) \mathrm{d} t=r \sin (\nu(t)+\omega) \sin i+C t_{\mathrm{e}}$ où

$r=\frac{a\left(1-e^{2}\right)}{1+e \cos \nu(t)}$ et $a \sin i=\frac{P K\left(1-e^{2}\right)^{1 / 2}}{2 \pi}$

on peut ainsi calculer $Z$ à chaque instant avec

$Z(t)=\frac{P K\left(1-e^{2}\right)^{3 / 2} \sin (\nu(t)+\omega)}{2 \pi(1+e \cos \nu(t))}+C t_{\mathrm{e}}$. 
Tableau 2. Paramètres d'ajustement de la variation de vitesse radiale. La période est exprimée en jours, $V_{\mathrm{o}}, K$ et $\sigma$ en km s ${ }^{-1}$, $\omega$ en radians. nvr et $m$ sont respectivement le nombre de vitesses utilisées dans le calcul et le nombre de mouvements nécessaires à la représentation de la pulsation. $\sigma$ est le résidu quadratique moyen de l'ajustement. Pour chaque étoile les lignes sont relatives respectivement au premier, second et troisième mouvement. L'astérisque indique que la période utilisée est celle de la photométrie.

\begin{tabular}{|c|c|c|c|c|c|c|c|c|c|c|c|c|c|c|}
\hline & Période & $n v r$ & $m$ & $\sigma$ & & & $V_{\mathrm{o}}$ & & $e$ & & $\omega$ & & & $K$ \\
\hline BL Her & $1,3074372 \pm 0,000007$ & 113 & 3 & 0,82 & 9,85 & \pm & 0,11 & $\begin{array}{l}0,471 \\
0,739 \\
0,457\end{array}$ & $\begin{array}{ll} \pm & 0,027 \\
\pm & 0,017 \\
\pm & 0,053\end{array}$ & $\begin{array}{l}1,36 \\
2,07 \\
1,77\end{array}$ & $\begin{array}{l} \pm 1 \\
\pm 0 \\
\pm\end{array}$ & $\begin{array}{l}0,12 \\
0,16 \\
0,24\end{array}$ & $\begin{array}{r}16,6 \\
13,5 \\
4,2\end{array}$ & $\begin{array}{l} \pm 1,4 \\
\pm 1,5 \\
\pm \quad 0,4\end{array}$ \\
\hline UY Mon & $2,398255 \pm 0,000007$ & 30 & 1 & 0,47 & 34,09 & \pm & 0,10 & 0,301 & $\pm 0,014$ & 1,84 & \pm 0 & 0,05 & 9,7 & $\pm 0,1$ \\
\hline ST Tau & $4,034299(*)$ & 32 & 1 & 0,73 & 1,11 & \pm & 0,15 & 0,412 & $\pm 0,011$ & 1,67 & \pm 0 & 0,03 & 18,0 & $\pm 0,2$ \\
\hline SY Cas & $4,071098(*)$ & 43 & 1 & 0,79 & $-46,93$ & \pm & 0,13 & 0,408 & $\pm 0,008$ & 1,67 & \pm 0 & 0,03 & 18,2 & $\pm 0,2$ \\
\hline Y Lac & $4,323776(*)$ & 43 & 1 & 1,12 & $-22,25$ & \pm & 0,19 & 0,388 & $\pm 0,012$ & 1,63 & \pm 0 & 0,04 & 18,6 & $\pm 0,3$ \\
\hline V402 Cyg & $4,364836(*)$ & 32 & 1 & 0,68 & $-13,42$ & \pm & 0,14 & 0,351 & $\pm 0,011$ & 1,53 & \pm 0 & 0,04 & 15,2 & $\pm 0,2$ \\
\hline V1154 Cyg & $4,92546(*)$ & 32 & 1 & 0,57 & $-4,32$ & \pm & 0,11 & 0,295 & $\pm 0,012$ & 1,55 & $\pm c$ & 0,05 & 12,7 & $\pm 0,2$ \\
\hline AS Per & $4,972516(*)$ & 20 & 1 & 0,55 & $-30,39$ & \pm & 0,16 & 0,473 & $\pm 0,008$ & 1,55 & $\pm c$ & 0,03 & 19,9 & $\pm 0,2$ \\
\hline BG Lac & $5,331932(*)$ & 31 & 2 & 0,34 & $-18,02$ & \pm & 0,07 & $\begin{array}{l}0,415 \\
0,133\end{array}$ & $\begin{array}{ll} \pm & 0,043 \\
\pm & 0,146\end{array}$ & $\begin{array}{l}0,86 \\
2,37\end{array}$ & $\begin{array}{l} \pm 0 \\
\pm 0\end{array}$ & $\begin{array}{l}0,41 \\
0,49\end{array}$ & $\begin{array}{r}17,1 \\
5,6\end{array}$ & $\begin{array}{l} \pm 4,9 \\
\pm \quad 4,7\end{array}$ \\
\hline RR Lac & $6,41629 \pm 0,00002$ & 29 & 2 & 0,39 & $-38,73$ & \pm & 0,10 & $\begin{array}{l}0,435 \\
0,351\end{array}$ & $\begin{array}{ll} \pm & 0,013 \\
\pm & 0,216\end{array}$ & $\begin{array}{l}1,30 \\
0,71\end{array}$ & $\begin{array}{l} \pm 0 \\
\pm 0\end{array}$ & $\begin{array}{l}0,06 \\
0,98\end{array}$ & $\begin{array}{r}17,3 \\
1,3\end{array}$ & $\begin{array}{ll} \pm & 0,5 \\
\pm & 0,5\end{array}$ \\
\hline BB Her & $7,50585 \pm 0,00008$ & 25 & 2 & 0,53 & 88,75 & \pm & 0,14 & $\begin{array}{l}0,371 \\
0,513\end{array}$ & $\begin{array}{ll} \pm & 0,018 \\
\pm & 0,101\end{array}$ & $\begin{array}{l}1,26 \\
2,21\end{array}$ & $\begin{array}{l} \pm 0 \\
\pm 0\end{array}$ & $\begin{array}{l}0,08 \\
0,46\end{array}$ & $\begin{array}{r}17,3 \\
2,9\end{array}$ & $\begin{array}{ll} \pm & 0,5 \\
\pm & 0,6\end{array}$ \\
\hline RS Ori & $7,566881(*)$ & 38 & 2 & 0,67 & 42,89 & \pm & 0,14 & $\begin{array}{l}0,436 \\
0,524\end{array}$ & $\begin{array}{ll} \pm & 0,012 \\
\pm & 0,068\end{array}$ & $\begin{array}{l}1,34 \\
2,36\end{array}$ & $\begin{array}{l} \pm 0 \\
\pm 0\end{array}$ & $\begin{array}{l}0,05 \\
0,24\end{array}$ & $\begin{array}{r}20,6 \\
3,8\end{array}$ & $\begin{array}{ll} \pm & 0,4 \\
\pm & 0,4\end{array}$ \\
\hline W Gem & $7,91343 \pm 0,00004$ & 48 & 2 & 0,68 & 1,61 & \pm & 0,11 & $\begin{array}{l}0,453 \\
0,591\end{array}$ & $\begin{array}{ll} \pm & 0,010 \\
\pm & 0,062\end{array}$ & $\begin{array}{l}1,22 \\
2,30\end{array}$ & $\begin{array}{l} \pm 0 \\
\pm 0\end{array}$ & $\begin{array}{l}0,03 \\
0,18\end{array}$ & $\begin{array}{r}21,0 \\
4,0\end{array}$ & $\begin{array}{l} \pm \quad 0,3 \\
\pm \quad 0,3\end{array}$ \\
\hline GQ Ori & $8,61645 \pm 0,00007$ & 29 & 2 & 0,50 & 45,12 & \pm & 0,13 & $\begin{array}{l}0,398 \\
0,362\end{array}$ & $\begin{array}{l} \pm 0,015 \\
\pm 0,065\end{array}$ & $\begin{array}{l}1,40 \\
2,64\end{array}$ & $\begin{array}{l} \pm 0 \\
\pm 0\end{array}$ & $\begin{array}{l}0,08 \\
0,32\end{array}$ & $\begin{array}{r}20,1 \\
5,5\end{array}$ & $\begin{array}{l} \pm 0,7 \\
\pm 0,6\end{array}$ \\
\hline VX Per & $10,8866 \pm 0,0002$ & 58 & 2 & 0,64 & $-35,97$ & \pm & 0,10 & $\begin{array}{l}0,380 \\
0,412\end{array}$ & $\begin{array}{ll} \pm & 0,013 \\
\pm & 0,038\end{array}$ & $\begin{array}{l}1,40 \\
2,69\end{array}$ & $\begin{array}{l} \pm 0 \\
\pm 0\end{array}$ & $\begin{array}{l}0,07 \\
0,16\end{array}$ & $\begin{array}{r}16,6 \\
6,8\end{array}$ & $\begin{array}{ll} \pm & 0,5 \\
\pm & 0,4\end{array}$ \\
\hline AA Gem & $11,30334(*)$ & 38 & 2 & 0,60 & 18,76 & \pm & 0,12 & $\begin{array}{l}0,330 \\
0,384\end{array}$ & $\begin{array}{ll} \pm & 0,029 \\
\pm & 0,037\end{array}$ & $\begin{array}{l}1,50 \\
1,85\end{array}$ & $\begin{array}{l} \pm 0 \\
\pm 0\end{array}$ & $\begin{array}{l}0,18 \\
0,20\end{array}$ & $\begin{array}{l}14,2 \\
12,2\end{array}$ & $\begin{array}{l} \pm 1,2 \\
\pm \quad 1,2\end{array}$ \\
\hline RX Aur & $11,623537(*)$ & 25 & 2 & 0,38 & $-21,95$ & \pm & 0,11 & $\begin{array}{l}0,268 \\
0,405\end{array}$ & $\begin{array}{ll} \pm & 0,022 \\
\pm & 0,051\end{array}$ & $\begin{array}{l}1,27 \\
1,87\end{array}$ & $\begin{array}{l} \pm 0 \\
\pm 0\end{array}$ & $\begin{array}{l}0,13 \\
0,28\end{array}$ & $\begin{array}{r}15,6 \\
6,7\end{array}$ & $\begin{array}{l} \pm \quad 0,9 \\
\pm \quad 0,8\end{array}$ \\
\hline TT Aql & $13,754707\left(^{*}\right)$ & 27 & 2 & 0,62 & 3,55 & \pm & 0,15 & $\begin{array}{l}0,375 \\
0,528\end{array}$ & $\begin{array}{ll} \pm & 0,023 \\
\pm & 0,031\end{array}$ & $\begin{array}{l}1,92 \\
1,86\end{array}$ & $\begin{array}{l} \pm 0 \\
\pm 0\end{array}$ & $\begin{array}{l}0,11 \\
0,18\end{array}$ & $\begin{array}{l}20,4 \\
13,2\end{array}$ & $\begin{array}{l} \pm \quad 1,1 \\
\pm \quad 1,2\end{array}$ \\
\hline SZ Cyg & $15,11034(*)$ & 43 & 2 & 0,51 & $-11,39$ & \pm & 0,09 & $\begin{array}{l}0,515 \\
0,424\end{array}$ & $\begin{array}{ll} \pm & 0,018 \\
\pm & 0,022\end{array}$ & $\begin{array}{l}1,27 \\
1,79\end{array}$ & $\begin{array}{l} \pm 0 \\
\pm 0\end{array}$ & $\begin{array}{l}0,11 \\
0,12\end{array}$ & $\begin{array}{l}19,1 \\
16,3\end{array}$ & $\begin{array}{l} \pm 1,2 \\
\pm \quad 1,2\end{array}$ \\
\hline SV Mon & $15,2342 \pm 0,0001$ & 40 & 2 & 0,56 & 27,32 & + & 0,11 & $\begin{array}{l}0,481 \\
0,554\end{array}$ & $\begin{array}{ll} \pm & 0,013 \\
\pm & 0,025\end{array}$ & $\begin{array}{l}1,63 \\
1,89\end{array}$ & $\begin{array}{l} \pm 0 \\
\pm 0\end{array}$ & $\begin{array}{l}0,06 \\
0,13\end{array}$ & $\begin{array}{l}23,8 \\
14,8\end{array}$ & $\begin{array}{l} \pm 1,0 \\
\pm 0,7\end{array}$ \\
\hline CD Cyg & $17,07414\left(^{*}\right)$ & 35 & 2 & 0,78 & $-11,45$ & \pm & 0,16 & $\begin{array}{l}0,445 \\
0,546\end{array}$ & $\begin{array}{ll} \pm & 0,023 \\
\pm & 0,036\end{array}$ & $\begin{array}{l}1,62 \\
1,90\end{array}$ & $\begin{array}{l} \pm 0 \\
\pm 0\end{array}$ & $\begin{array}{l}0,12 \\
0,22\end{array}$ & $\begin{array}{l}23,4 \\
13,8\end{array}$ & $\begin{array}{l} \pm 1,5 \\
\pm \quad 1,6\end{array}$ \\
\hline SV Vul & $45,055 \pm 0,002$ & 41 & 2 & 0,36 & $-2,01$ & I & 0,07 & $\begin{array}{l}0,386 \\
0,589\end{array}$ & $\begin{array}{ll} \pm & 0,017 \\
\pm & 0,089\end{array}$ & $\begin{array}{l}1,68 \\
4,85\end{array}$ & $\begin{array}{l} \pm 0 \\
\pm 0\end{array}$ & $\begin{array}{l}0,10 \\
0,92\end{array}$ & $\begin{array}{r}25,3 \\
4,1\end{array}$ & $\begin{array}{l} \pm 2,2 \\
\pm \quad 2,2\end{array}$ \\
\hline
\end{tabular}

Si $P$ est exprimé en jours, $K$ en $\mathrm{km} \mathrm{s}^{-1}, Z(t)$ s'exprime en rayons solaires par

$$
Z(t)=0,019757 \frac{P K\left(1-e^{2}\right)^{3 / 2} \sin (\nu(t)+\omega)}{(1+e \cos \nu(t))}+C t_{\mathrm{e}} .
$$

Pour $m$ mouvements, on peut alors calculer $\Delta R$ dans le cas général avec

$$
\Delta R=\alpha\left|\sum_{m}\left[Z_{m}\left(t_{1 m}\right)-Z_{m}\left(t_{2 m}\right)\right]\right| .
$$

Pour chacune des étoiles nous avons ainsi calculé les amplitudes de variation des rayons en utilisant les paramètres des ajustements képlériens. Les résultats de ces calculs sont donnés dans le tableau 3.

\subsection{Calcul des rayons}

Pour l'époque $t$ le rayon de l'étoile est calculé avec $R(t)=-\alpha \sum_{m} Z_{m}(t)+R_{\mathrm{c}}$

où $R_{\mathrm{c}}$ est un rayon qui sera pris pour référence dans les calculs. Dans le cas d'un seul mouvement, ce rayon est le rayon d'équilibre correspondant à une accélération nulle. Nous avons montré (Imbert 1987), qu'à toutes les phases de la pulsation on peut écrire une relation liant le rayon de l'étoile, la magnitude et un indice de couleur. Avec les couleurs $V$ et $B$, on peut écrire :

$V(t)=-5 \log R(t)-a(B-V)(t)+Q$ 
Tableau 3. Dimensions des 22 céphéides. $R_{\max }, R_{\mathrm{o}}$ et $\Delta R$ sont exprimés en rayons solaires. $n b v$ est le nombre de mesures $B$ et $V$ utilisées pour le calcul des rayons. $a$ est le coefficient de proportionnalité entre $10 \log T_{\mathrm{e}}+C B$ et $B-V$

\begin{tabular}{|c|c|c|c|c|c|c|}
\hline & Période & $n b v$ & $R_{\max }$ & $R_{\mathrm{O}}$ & $\Delta R$ & $a$ \\
\hline BL Her & 1,31 & 69 & 12,3 & $11,7 \pm 0,9$ & $1,24 \pm 0,07$ & $2,48 \pm 0,05$ \\
\hline UY Mon & 2,40 & 25 & 35,9 & $35,3 \pm 8,6$ & $1,18 \pm 0,01$ & $2,56 \pm 0,11$ \\
\hline ST Tau & 4,03 & 55 & 46,3 & $44,7 \pm 2,4$ & $3,55 \pm 0,03$ & $2,29 \pm 0,03$ \\
\hline SY Cas & 4,07 & 96 & 50,9 & $49,3 \pm 5,5$ & $3,63 \pm 0,03$ & $2,26 \pm 0,05$ \\
\hline Y Lac & 4,32 & 34 & 56,0 & $54,2 \pm 5,7$ & $3,98 \pm 0,04$ & $2,33 \pm 0,05$ \\
\hline V402 Cyg & 4,36 & 85 & 47,9 & $46,4 \pm 6,5$ & $3,33 \pm 0,03$ & $1,96 \pm 0,08$ \\
\hline V1154 Cyg & 4,93 & 93 & 53,9 & $52,5 \pm 4,8$ & $3,22 \pm 0,03$ & $2,01 \pm 0,06$ \\
\hline AS Per & 4,97 & 44 & 54,1 & $52,1 \pm 4,8$ & $4,67 \pm 0,04$ & $2,44 \pm 0,05$ \\
\hline BG Lac & 5,33 & 84 & 50,7 & $48,8 \pm 5,3$ & $4,30 \pm 0,03$ & $2,21 \pm 0,07$ \\
\hline RR Lac & 6,42 & 192 & 63,9 & $61,6 \pm 3,1$ & $5,19 \pm 0,04$ & $2,17 \pm 0,03$ \\
\hline BB Her & 7,51 & 26 & 65,5 & $63,0 \pm 4,7$ & $5,62 \pm 0,21$ & $2,11 \pm 0,05$ \\
\hline RS Ori & 7,57 & 50 & 81,0 & $78,1 \pm 4,9$ & $6,60 \pm 0,17$ & $2,12 \pm 0,03$ \\
\hline W Gem & 7,91 & 60 & 66,9 & $64,2 \pm 8,2$ & $6,55 \pm 0,12$ & $1,95 \pm 0,07$ \\
\hline GQ Ori & 8,62 & 49 & 77,2 & $73,8 \pm 3,5$ & $7,43 \pm 0,41$ & $2,01 \pm 0,03$ \\
\hline VX Per & 10,89 & 62 & 95,7 & $91,1 \pm 6,2$ & $8,98 \pm 0,32$ & $2,19 \pm 0,04$ \\
\hline AA Gem & 11,30 & 90 & 93,5 & $87,7 \pm 5,6$ & $10,19 \pm 0,78$ & $1,90 \pm 0,05$ \\
\hline RX Aur & 11,62 & 45 & 96,1 & $91,2 \pm 7,9$ & $9,25 \pm 0,58$ & $1,98 \pm 0,05$ \\
\hline TT Aql & 13,75 & 60 & 100,5 & $91,4 \pm 4,9$ & $16,7 \pm 0,8$ & $2,19 \pm 0,04$ \\
\hline SZ Cyg & 15,11 & 48 & 106,8 & $97,4 \pm 5,6$ & $17,7 \pm 0,9$ & $2,18 \pm 0,05$ \\
\hline SV Mon & 15,23 & 192 & 111,6 & $100,8 \pm 3,7$ & $20,2 \pm 0,7$ & $2,11 \pm 0,03$ \\
\hline CD Cyg & 17,07 & 121 & 125,7 & $113,8 \pm 4,1$ & $22,7 \pm 1,3$ & $2,13 \pm 0,03$ \\
\hline SV Vul & 45,06 & 113 & 238,4 & $216,5 \pm 6,8$ & $50,5 \pm 3,6$ & $2,11 \pm 0,03$ \\
\hline
\end{tabular}

$a$ est le coefficient de proportionnalité entre $10 \log T_{\mathrm{e}}+$ CB et $B-V$ (Imbert 1987). En utilisant le déplacement

$D(t)=-\alpha \sum_{m} Z_{m}(t)$

on aura

$V(t)=-5 \log \left[D(t)+R_{\mathrm{c}}\right]-a(B-V)(t)+Q$.

Pour chacune des époques des nbv observations de $V$ et $B-V$ on calcule $D(t)$. On dispose ainsi de $n b v$ équations dont les inconnues à trouver sont $a, Q$ et $R_{\mathrm{c}}$. Dans un premier calcul, on détermine une solution par moindres carrés en linéarisant l'Éq. (1). En négligeant $D(t) / R_{\mathrm{c}}^{2}$ on est conduit à

$V(t)=-2,1715 D(t) / R_{\mathrm{c}}-a(B-V)(t)+Q^{\prime}$.

À partir de cette solution approchée, on détermine les vraies valeurs en résolvant par approximations successives les équations de la forme

$V_{\text {obs }}(t)-V_{\text {app }}(t)=-\frac{2,1715}{R_{\mathrm{c}}-D(t)} \mathrm{d} R_{\mathrm{c}}-(B-V)(t) \mathrm{d} a+\mathrm{d} Q$

où $V_{\text {obs }}(t)-V_{\text {app }}$ sont respectivement la vitesse observée et la vitesse calculée. Après la détermination de $R_{\mathrm{c}}$ il reste à formuler la différence entre le rayon moyen de l'étoile, $R_{\mathrm{o}}$, et $R_{\mathrm{c}}$. Le rayon moyen $R_{\mathrm{o}}$ est défini par

$R_{\mathrm{o}}=\frac{1}{P_{0}} \int_{0}^{P_{0}} R(t) \mathrm{d} t+R_{\mathrm{c}}$.
Dans cette expression $P_{\mathrm{o}}$ correspond au mouvement de plus longue période. Nous avons montré (Imbert 1987), que pour un seul mouvement, avec les unités utilisée plus hauts,

$R_{\mathrm{o}}=0,029636$ e K P $\left(1-e^{2}\right)^{1 / 2} \sin \omega+R_{\mathrm{c}}$.

L'intégration étant linéaire, ce résultat se généralise facilement au cas de plusieurs mouvements. On a ainsi :

$R_{\mathrm{o}}=R_{\mathrm{c}}+0,029636 \sum_{m} e_{m} K_{m} P_{m}\left(1-e_{m}^{2}\right)^{1 / 2} \sin \omega_{m}$.

Pour la détermination des rayons, nous avons utilisé la photométrie $U B V$ car, pour les étoiles mesurées, cette photométrie réalise le meilleur compromis entre la qualité des mesures et la proximité des observations avec nos mesures de vitesses radiales, condition essentielle pour ne pas biaiser les calculs par une éventuelle dérive de la période. De plus, pour la plupart de nos étoiles, le nombre de mesures dans les couleurs $R, I, J$ ou $K$ est très insuffisant pour la détermination des rayons. Les résultats de ces calculs figurent dans le tableau 3. Sur cet échantillon homogène mais partiel, car pour l'instant la zone de période 17 à 45 jours n'est pas encore couverte, nous avons calculé une relation linéaire liant $\log P$ et $\log R_{\mathrm{o}}$.

$\log R_{\mathrm{o}}=0,611 \pm 0,023 \log P+1,296 \pm 0,022$.

BL Her, d'un type particulier, n'a pas été considérée dans ce calcul. La relation entre $\log P$ et $\log R_{\mathrm{o}}$ est représentée sur la Fig. 1. Ce résultat est très proche, notamment, de 

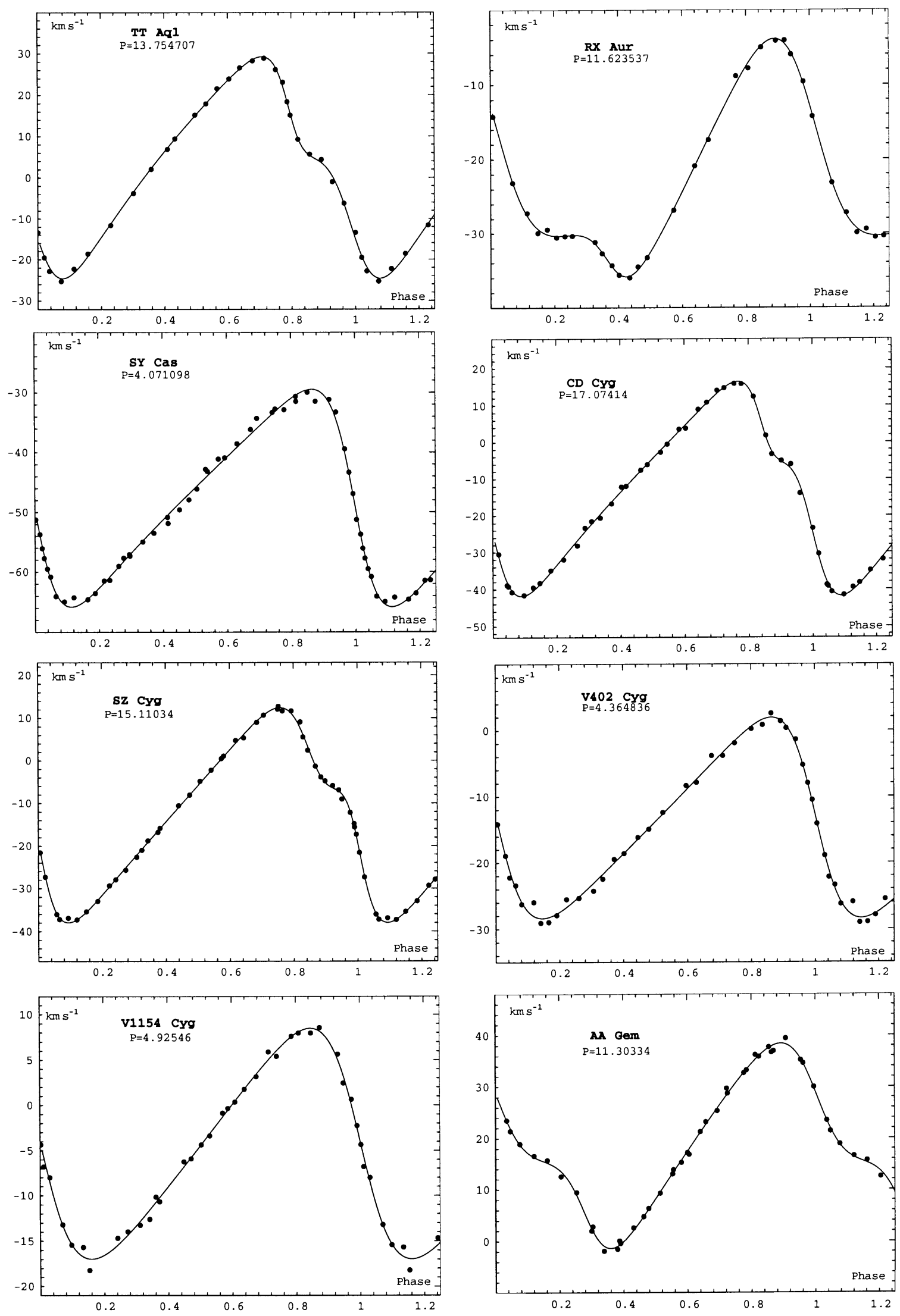

Fig. 2. Vitesses radiales et ajustement par mouvements képlériens fictifs de TT Aql, RX Aur, SY Cas, CD Cyg, SZ Cyg, V402 Cyg, V1154 Cyg et AA Gem 

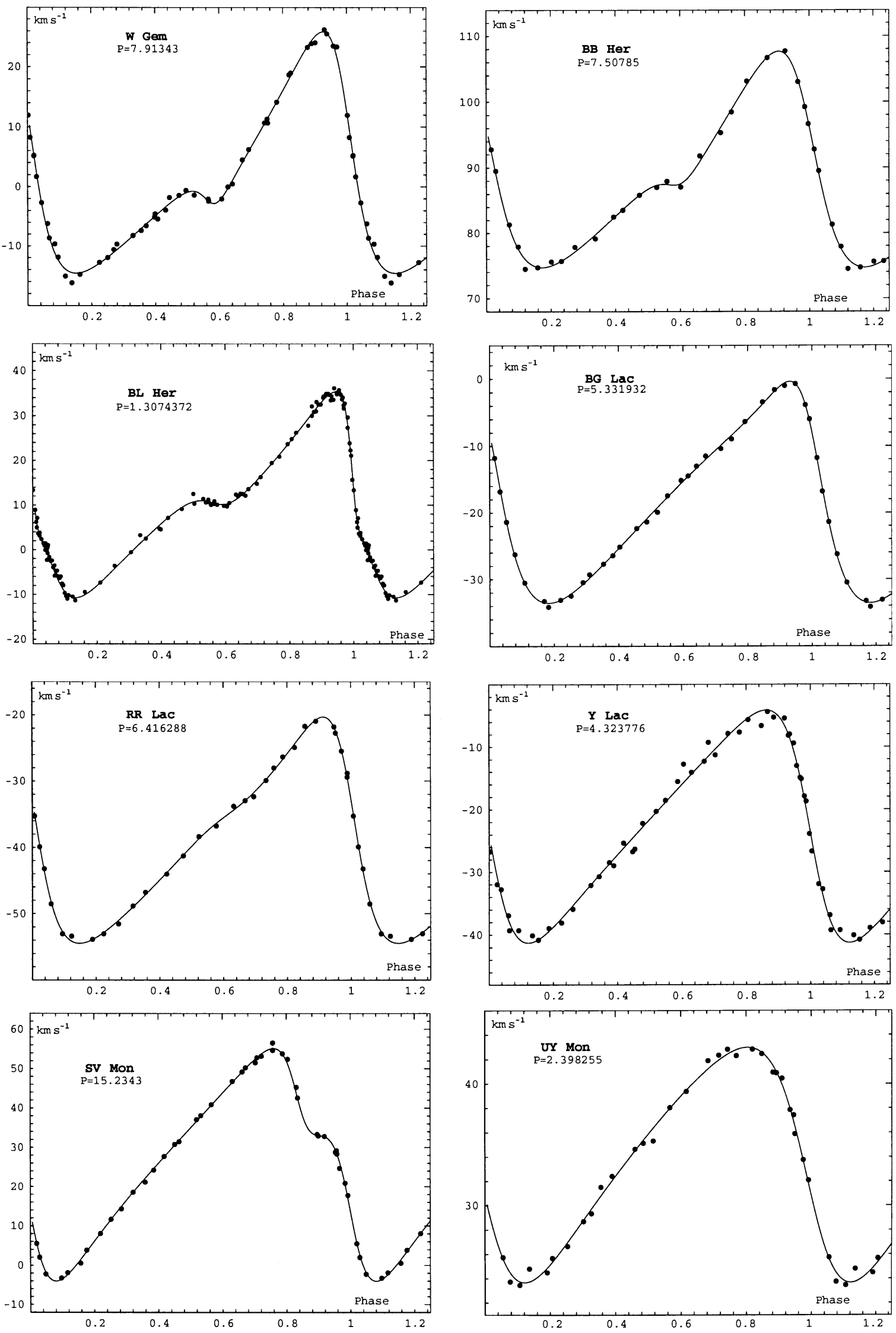

Fig. 3. Vitesses radiales et ajustement par mouvements képlériens fictifs de W Gem, BB Her, BL Her, BG Lac, RR Lac, Y Lac, SV Mon et UY Mon 

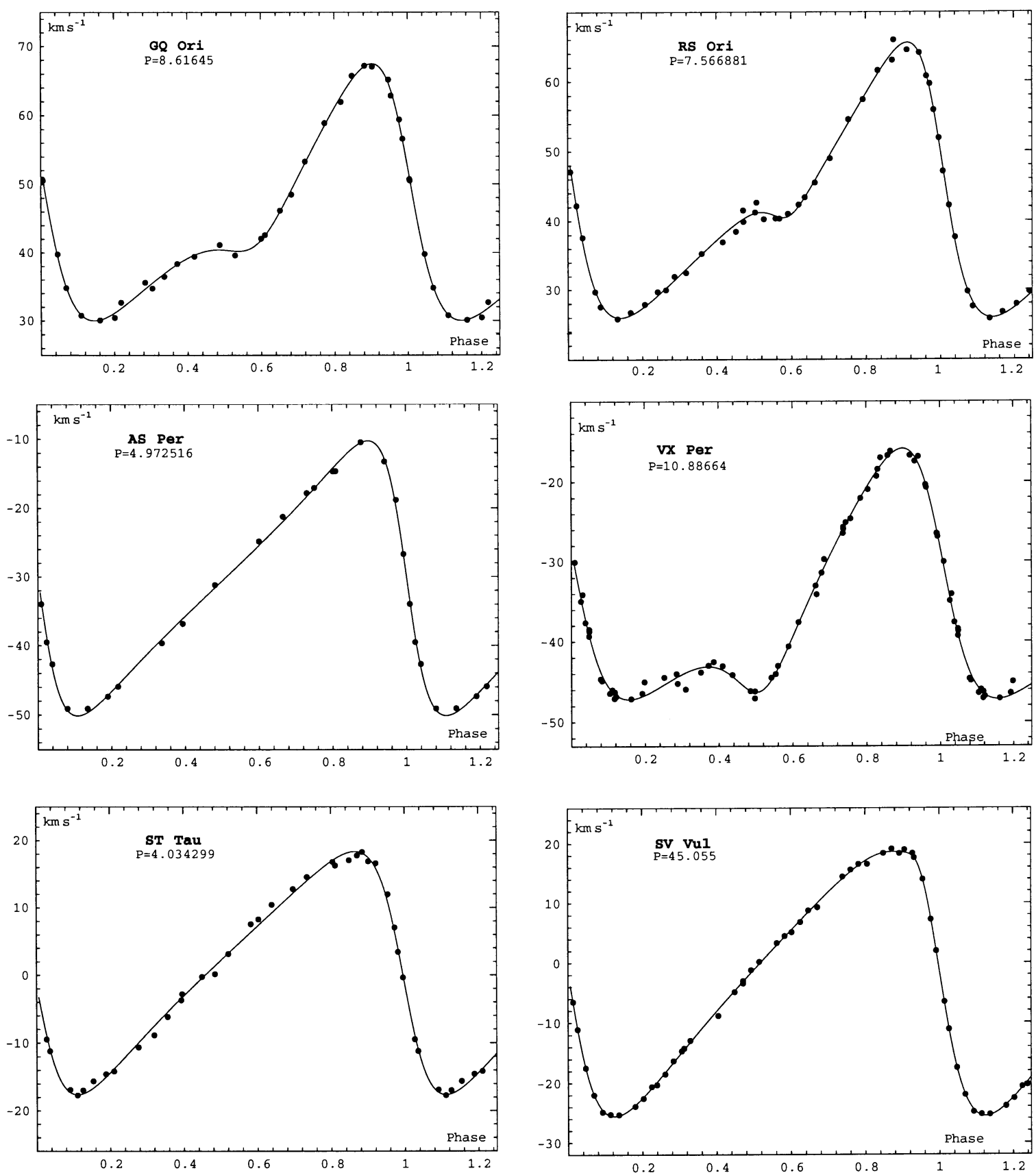

Fig. 4. Vitesses radiales et ajustement par mouvements képlériens fictifs de GQ Ori, RS Ori, AS Per, VX Per, ST Tau et SV Vul

celui obtenu, avec $V$ et $B-V$ par Laney \& Stobie (1995), qui donnent

$\log R_{\mathrm{o}}=0,625 \pm 0,031 \log P+1,198 \pm 0,010$

Cependant il y a désaccord avec les déterminations déduites d'autres couleur, notamment $J$ et $K$. Il semble que les couleurs $J$ et $K$ soient moins sensibles aux effets de variations de gravité ou de microturbulence au cours de la pulsation. Laney \& Stobie (1995) donnent une formule de correction au premier ordre pour tenir compte de la variation de gravité. Si nous appliquons ces corrections à nos rayons nous déduisons la formulation suivante pour la relation période rayon :

$\log R_{\mathrm{o}}=0,648 \pm 0,025 \log P+1,298 \pm 0,022$.

Les coefficients restent néanmoins assez différents de ceux de Laney et Stobie pour les couleurs $J$ et $K$. Cette différence doit, sans doute, être attribuée aux autres effets non pris en compte, tels que microturbulence ou duplicité. On peut aussi se demander si avec les couleurs $B$ et $V$, le coefficient de projection et l'assombrissement centre-bord ne varient pas de manière plus significative au cours de 
la pulsation, et biaisent ainsi la détermination du rayon moyen.

\section{Remarques individuelles}

Sauf indication contraire la période retenue pour les calculs est celle de la photométrie donnée dans la base de Fernie et al. (1995). La période déterminée avec les vitesses radiales étant en accord avec cette valeur. Dans les cas où la période photométrique ne convient pas pour représenter nos vitesses radiales, nous avons déterminé celle-ci en laissant le paramètre $P$, libre dans le calcul des éléments. Les calculs ont été faits en utilisant les couleurs $B$ et $V$ dont les mesures sont plus nombreuses et plus homogènes que pour les autres indices de couleur. L'ajustement des observations de vitesses radiales est généralement fait avec un seul mouvement pour les périodes inférieures à 5 jours et avec deux mouvements pour les périodes plus longues que 5 jours. Dans tous les cas où plusieurs mouvements sont nécessaires, le meilleur ajustement est obtenu en donnant la même valeur de la période à tous les mouvements plutôt que des sous-multiples de celle-ci.

TT Aql. Deux mouvements de même période sont nécessaires pour représenter les observations de vitesses radiales. La photométrie utilisée est celle donnée par Berdnikov (1993, 1992b, 1992e).

RX Aur. Deux mouvements sont nécessaires à la représentation des observations de vitesses radiales. La photométrie est celle de Berdnikov (1986, 1992b).

SY Cas. Un seul mouvement suffit pour représenter les observations de vitesses radiales. La photométrie utilisée est celle donnée par Berdnikov (1992a, 1992b, 1992c).

SZ Cyg. Deux mouvements de même période représentent très bien les observations de vitesses radiales. La photométrie est celle de Berdnikov (1986, 1992b).

CD Cyg. Deux mouvements de même période représentent très bien les observations de vitesses radiales malgré l'insuffisance de mesures aux alentours du "bump". La photométrie utilisée est celle de Berdnikov (1986, 1992e, 1993).

V402 Cyg. Un seul mouvement est suffisant pour représenter les observations de vitesses radiales. La photométrie utilisée est celle donnée par Moffett \& Barnes (1984) et Berdnikov (1992a, 1993).

V1154 Cyg. Un seul mouvement représente très bien les observations de vitesses radiales. La photométrie est celle de Berdnikov (1987, 1992c, 1993).

W Gem. La période utilisée est celle redéterminée avec nos vitesses radiales, $P=7,913430 \pm 0,000036$ jours. Deux mouvements de même période sont nécessaires pour représenter les observations de vitesses radiales. La photométrie retenue est celle donnée par Szabados (1980), Moffett \& Barnes (1984) et Kiss (1998).

AA Gem. Deux mouvements de même période représentent très bien les observations de vitesses radiales malgré la faible amplitude du "bump". La photométrie utilisée est celle donnée par Moffett \& Barnes (1984) et Berdnikov (1986, 1992b, 1992e).

BB Her. La période utilisée est celle déterminée avec nos vitesses radiales, $P=7,507854 \pm 0,000078$ jours. Deux mouvements de même période représentent très bien les observations de vitesses radiales. La photométrie utilisée est celle de Berdnikov (1992a).

BL Her. Cette céphéide est d'un type particulier. Pour représenter correctement les observations nous avons utilisé trois mouvements de même période. Le calcul de la période, en utilisant uniquement les vitesses radiales nous a donné $P=1,3074372 \pm 0,0000006$ jour. Les $U B V$ utilisés sont ceux donnés par Moffett \& Barnes (1984).

Y Lac. Un seul mouvement suffit pour représenter les observations de vitesses radiales. Cependant le $\mathrm{O}-\mathrm{C}$ moyen des résidus laissé par cet ajustement $\left(1,12 \mathrm{~km} \mathrm{~s}^{-1}\right)$ est sensiblement plus grand que l'erreur moyenne sur la détermination des vitesses $(0,75)$. L'ajout d'un deuxième mouvement n'améliore pas sensiblement l' ajustement. La photométrie utilisée est celle de Moffett \& Barnes (1984).

RR Lac. La période utilisée est celle déterminée avec nos vitesses radiales, $P=6,41629 \pm 0,00002$ jours. Les observations sont sensiblement mieux représentées par deux mouvements que par un seul. Le $\sigma$ des résidus passant de 0,57 à $0,39 \mathrm{~km} \mathrm{~s}^{-1}$, si on utilise deux mouvements. La photométrie est celle de Berdnikov (1992a, 1992c, 1992d, 1992e, 1993) et Berdnikov \& Voziakova (1995).

BG Lac. Dans un premier calcul, un seul mouvement a été utilisé pour l'ajustement, mais les observations sont mieux représentées par deux mouvements que par un seul. Le $\sigma$ des résidus passant de 0,53 à $0,34 \mathrm{~km} \mathrm{~s}^{-1}$, si on utilise deux mouvements. La photométrie utilisée est celle donnée par Szabados (1980), Moffett \& Barnes (1984), Berdnikov (1992a) et Berdnikov \& Voziakova (1995).

SV Mon. La période photométrique donnée dans la base de Fernie et al. (1995), 15,23278 jours, n'est pas satisfaisante pour nos observations de vitesses. Nous avons redéterminé sa valeur à $15,2343 \pm 0,0001$ jours. Deux mouvements de même période représentent très bien les observations de vitesses radiales. La photométrie utilisée est celle donnée par Szabados (1981), Moffett \& Barnes (1984), Coulson \& Caldwell (1985), Berdnikov (1986, 1992b, 1992e, 1993), Berdnikov \& Yakubov (1993), Berdnikov \& Turner (1995) et Berdnikov \& Voziakova (1995).

UY Mon. La nature Céphéide de cette étoile a été démontrée dans un travail précédent (Imbert 1981b). Nous avions alors calculé une période 2,39813 jours. La base de Fernie et al. mentionne 2,39797, valeur qui ne représente pas correctement nos vitesses. Nous avons redéterminé cette période et avons obtenu 2,398255 \pm 0,000008 jours, valeur en accord avec notre première détermination. Un seul mouvement suffit pour représenter les observations de vitesses radiales. La photométrie 
utilisée est celle donnée par Berdnikov \& Turner (1995) et Berdnikov \& Voziakova (1995). L'erreur, relativement grande, sur le rayon est due au petit nombre de mesures $U B V$ disponibles.

RS Ori. Deux mouvements de même période représentent très bien les observations de vitesses radiales. La photométrie utilisée est celle donnée par Moffett \& Barnes (1984) et Szabados (1991).

GQ Ori. La période utilisée est celle déterminée avec nos vitesses radiales, $P=8,61645 \pm 0,00007$ jours. Deux mouvements de même période sont nécessaires pour représenter les observations de vitesses radiales. La photométrie utilisée est celle de Moffett \& Barnes (1984).

VX Per. La période utilisée est celle déterminée avec nos vitesses radiales, $P=8,88662 \pm 0,00024$ jours. Cette valeur est sensiblement différente de celle de la base de Fernie et al. (10,88904). Deux mouvements de même période représentent très bien les observations de vitesses radiales. La photométrie retenue est celle donnée par Berdnikov (1987, 1992e), Berdnikov \& Yakubov (1993).

AS Per. Un seul mouvement suffit pour représenter les observations de vitesses radiales. La photométrie utilisée est celle de Moffett \& Barnes (1984).

ST Tau. Un seul mouvement suffit pour représenter les observations de vitesses radiales. La photométrie utilisée est celle de Moffett \& Barnes (1984).

SV Vul. Le meilleur ajustement des vitesses radiales est obtenus avec deux mouvements. La période de Fernie et al. (1995), 44,944772 jours est différente de celle que nous avons déterminée à partir de nos vitesses. Nous avons obtenu 45,0548 \pm 0,0024 jours. La photométrie utilisée est celle donnée par Moffett \& Barnes (1984), Berdnikov (1986, 1987).

Remerciements. Quelques observations de vitesses radiales ont été obtenues grâce à la collaboration de C. Bardin et L. Prévot que je remercie cordialement.

\section{Bibliographie}

Baranne A., Mayor M., Poncet J.L., 1979, Vistas Astron. 23, 279

Bernikov L.N., 1986, Variables Stars 22, 369

Bernikov L.N., 1987, Variables Stars 22, 530

Bernikov L.N., 1992a, Astron. Astrophys. Trans. 2, 1

Bernikov L.N., 1992b, Astron. Astrophys. Trans. 2, 43

Bernikov L.N., 1992c, Astron. Astrophys. Trans. 2, 107

Bernikov L.N., 1992d, Astron. Astrophys. Trans. 2, 157

Bernikov L.N., 1992e, Pis'ma Astron. J. 18, 325

Bernikov L.N., 1993, Pis'ma Astron. J. 19, 210

Berdnikov L.N., Turner D.G., 1995, Pis'ma Astron. J. 21, 803

Bernikov L.N., Voziakova O.V., 1995, Pis'ma Astron. J. 21, No. 5

Berdnikov L.N., Yakubov S.D., 1993, Variable Stars 23, 47

Binnendijk L., 1960, Properties of double stars. University of Pennsylvania Press

Burki G., Mayor M., Benz W., 1982, A\&A 109, 258

Burki G., Benz W., 1982, A\&A 115, 30

Coulson I.M., Caldwell J.A.R., 1985, SAAO Circ. 9, 5

Fernie J.D., Beattie B., Evans N.R., Seager S., 1995, IBVS 4148

Imbert M., 1972, A\&A 18, 267

Imbert M., 1981a, A\&AS 44, 319

Imbert M., 1981b, IBVS 1983

Imbert M., Andersen J., Ardeberg A., et al., 1985, A\&AS 61, 259

Imbert M., 1987, A\&A 175, 30

Imbert M., Andersen J., Ardeberg A., et al., 1989, A\&AS 81, 339

Imbert M., 1994, A\&AS 105, 1

Imbert M., 1996, A\&AS 116, 497

Kiss L.L., 1998, MNRAS 297, 825

Laney C.D., Stobie R.S., 1995, MNRAS 274, 337

Lehman-Filhes R., 1894, Astron. Nachr. 136, 17

Moffett T.J., Barnes T.G., 1984, ApJS 55, 389

Schlesinger F., 1908, Allegh. Obs. Publ. 1, 33

Szabados L., 1980, Commun. Konkoly Obs. Hung Acad. Sci., Budapest, No. 76

Szabados L., 1981, Commun. Konkoly Obs. Hung Acad. Sci., Budapest, No. 77

Szabados L., 1991, Commun. Konkoly Obs. Hung Acad. Sci., Budapest, No. 96 